\title{
High energy gamma-ray study of the microquasar 1E 1740.7-2942 with Fermi-LAT
}

\section{Naoki Nishimura}

Department of Physical Sciences, Ritsumeikan University

\section{Masaki Mori*}

Department of Physical Sciences, Ritsumeikan University

E-mail: morimefc.ritsumei.ac.jp

The microquasar 1E 1740.7-2942, discovered by the Einstein satellite, is located near the Galactic Center at an angular distance of $50^{\prime}$ from Sgr A*, and the brightest X-ray source above 20 $\mathrm{keV}$ in the Galactic Center region. It has extended radio lobes reaching distances of up to a few parsecs and its core radio emission is variable. In X-ray energies it shows the spectral and timing properties similar to those of black hole candidates like Cyg X-1. GRANAT/SIGMA reported a burst of soft gamma-ray emission (300-600 keV) in 1990s which was interpreted as an electron-positron annihilation signal, but other satellite observations could not confirm the high energy feature reported by SIGMA, although a high energy tail extending up to $600 \mathrm{keV}$ with a power-law photon index of $1.9 \pm 0.1$ has been reported by INTEGRAL, indicating a non-thermal process which might accelerate particles to even higher energies. In this paper we report the result of gamma-ray study of 1E 1740.7-2942 above $100 \mathrm{MeV}$ using the six-year Fermi-LAT archival data, and its implication on particle acceleration process in microquasars is discussed.

The 34th International Cosmic Ray Conference,

30 July- 6 August, 2015

The Hague, The Netherlands

\footnotetext{
* Speaker.
} 


\section{Introduction}

The microquasar 1E 1740.7-2942, discovered by the Einstein satellite [1], is located near the Galactic Center at an angular distance of 50' from Sgr A*, and the brightest X-ray source above $20 \mathrm{keV}$ in the Galactic Center region [2]. It has extended radio lobes reaching distances of up to a few parsecs and its core radio emission is variable. In X-ray energies it shows the spectral and timing properties similar to those of black hole candidates like Cyg X-1 [3]. GRANAT/SIGMA reported a burst of soft gamma-ray emission (300-600 keV) in 1990s [4] which was interpreted as an electron-positron annihilation signal, but other satellite observations could not confirm the high energy feature reported by SIGMA, although a high energy tail extending up to $600 \mathrm{keV}$ with a power-law photon index of $-1.9 \pm 0.1$ has been reported by INTEGRAL [5], indicating a non-thermal process which might accelerate particles to even higher energies.

Recently, a persistent gamma-ray signal from SS433/W50 has been reported using the FermiLAT data [6] which may be the first microquasar detected at high-energy gamma-rays. This detection prompts us to search for other examples of gamma-ray emitting microquasar population.

In this paper we report the result of gamma-ray study of a microquasar 1E 1740.7-2942 above $100 \mathrm{MeV}$ using the six-year Fermi-LAT archival data, and its implication on particle acceleration process in microquasars is discussed.

\section{Analysis}

Six and a half years of archival data from Fermi/LAT have been analyzed using the Fermi Science Tools supplied by Fermi Science Support Center (FSSC [7], Ferm Science Tools v9r32p5). The energy range used in the present analysis was from $100 \mathrm{MeV}$ to $300 \mathrm{GeV}$. "Source" class events detected at zenith angles smaller than $100^{\circ}$ and within $30^{\circ}$ from 1E 1740.7-2942, located at $\left(\alpha_{2000}, \delta_{2000}\right)=\left(265.978^{\circ},-29.7452^{\circ}\right)$, were used for analysis, using the "P7REP_SOURCE_V15" instrument response function along with the standard analysis pipeline suggested by FSSC. The significance of the:gamma-ray signal has been estimated using a maximum likelihood method with the help of the gt like program (which we used in the binned mode) included in the tools. For the background model, we included 3EG sources «8] within $40^{\circ}$ from 1E 1740.7-2942, and the galactic diffuse emission and the isotropic diffuse component whose versions are those recommended in the instruction given by FSSC. The data periods for this studies span from 2008 August 4 to 2015 February 11.

\section{Results}

Fig 1 shows a gamma-ray countmap around the 1E 1740.7-2942 region. One may note a small excess around the nominal position of 1E 1740.7-2942. However, the test-statistic (TS) value for the position is 23.1 , just below the threshold to claim a source (normally $T S>25$ ). Since the Galactic diffuse model may not be perfect and there are many point sources near the Galactic center region which could affect the analysis, so we should be cautious to claim a new source unless the test-statistic is compelling. Thus, we computed an upper limit on the gamma-ray flux using the profile method as $7.8 \times 10^{-8} \mathrm{~cm}^{-2} \mathrm{~s}^{-1}$ (95\% C.L.) above $100 \mathrm{MeV}$. 


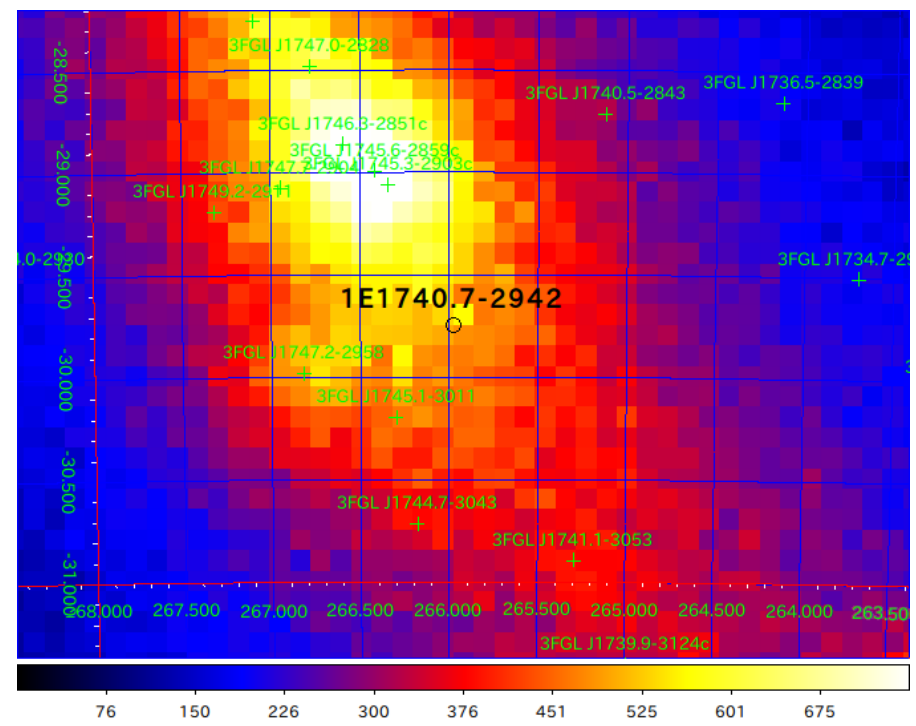

Figure 1: Gamma-ray countmap around the 1E 1740.7-2942 region. The map is created in $0^{\circ} .1$ grid for the data during 2008 August 4 to 2015 February 11. 3FGL sources are anotated.

The X-ray flux from 1E 1740.7-2942 observed by Swift/BAT shows long term variation over 2005 to 2014 [9], and we might also expect the gamma-ray flux is variable. Fig. 2] shows the X-ray light curve observed by Swift/BAT (upper panel) 9 and gamma-ray upper limits ( $>100 \mathrm{MeV}$, $90 \%$ C.L.) in half-year bins analyzed in the same way. No compelling evidence for gamma-ray signal is observed in any bin.

\section{Discussion}

Fig 3 shows the spectral energy distribution of 1E 1740.7-2942 compiled by ASDC [10] and from INTEGRAL/SPI [5] with our upper limit. In ASDC web Tools, a source appeared in the 1FGL catalog, 1FGL J1744.0-2931c [11], is assumed to be the counterpart (its angular separation is $0.21^{\circ}$ from $1 \mathrm{E} 1740.7-2942$ ), but there is no corresponding source in the 3FGL catalog [8] (probably it has disappeared because of the improved Galactic diffuse emission model [12]): thus we did not include the data points of 1FGL J1744.0-2931c here and plotted our upper limit only in the gamma-ray band. With our upper limit, the power-law spectrum with an index of $-1.9 \pm 0.1$ seen in the sub MeV band [5] should bend or cut below the $100 \mathrm{MeV}$ band, which may indicate the limit of particle acceleration process in 1E 1740.7-2942. If we join the point at $600 \mathrm{keV}$ and the limit at $100 \mathrm{MeV}$, the slope of the line is -0.7 which corresponds to a power-law of $E^{-2.7}$.

\section{Conclusion}

We have analyzed the six-year Fermi-LAT archival data to search for a gamma-ray emission from a microquasar 1E 1740.7-2942. The gamma-ray signal from this source is not significant and we obtained an upper limit on the gamma-ray flux of $7.8 \times 10^{-8} \mathrm{~cm}^{-2} \mathrm{~s}^{-1}$ (95\% C.L.) above $100 \mathrm{MeV}$. There is no indication of time variation of the gamma-ray flux in the half-year bin. 

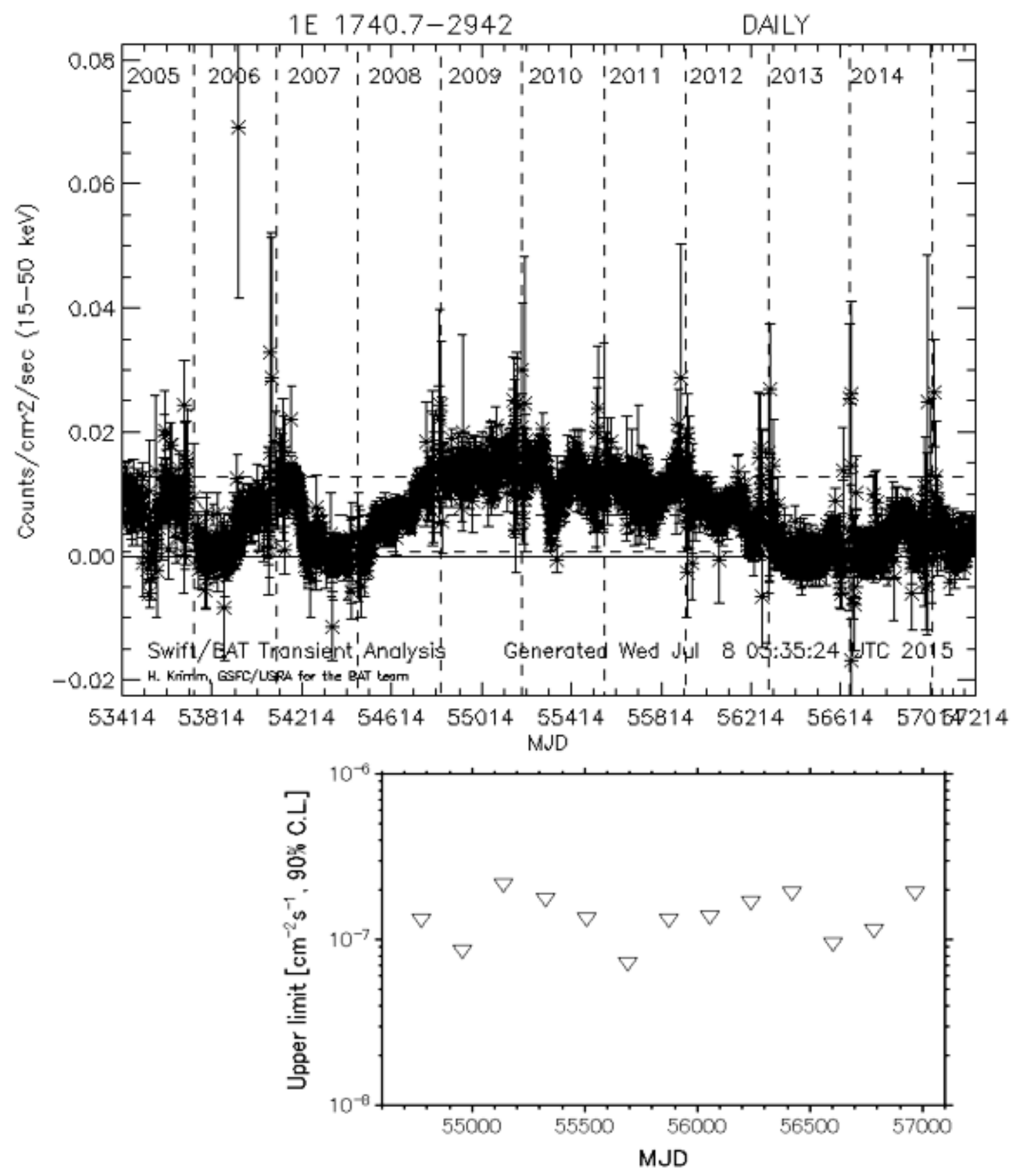

Figure 2: X-ray light curve observed by Swift/BAT (upper panel) [9] compared with gamma-ray upper limits (> $100 \mathrm{MeV}, 90 \%$ C.L.) in half-year bins (lower panel). Time axes are aligned. 


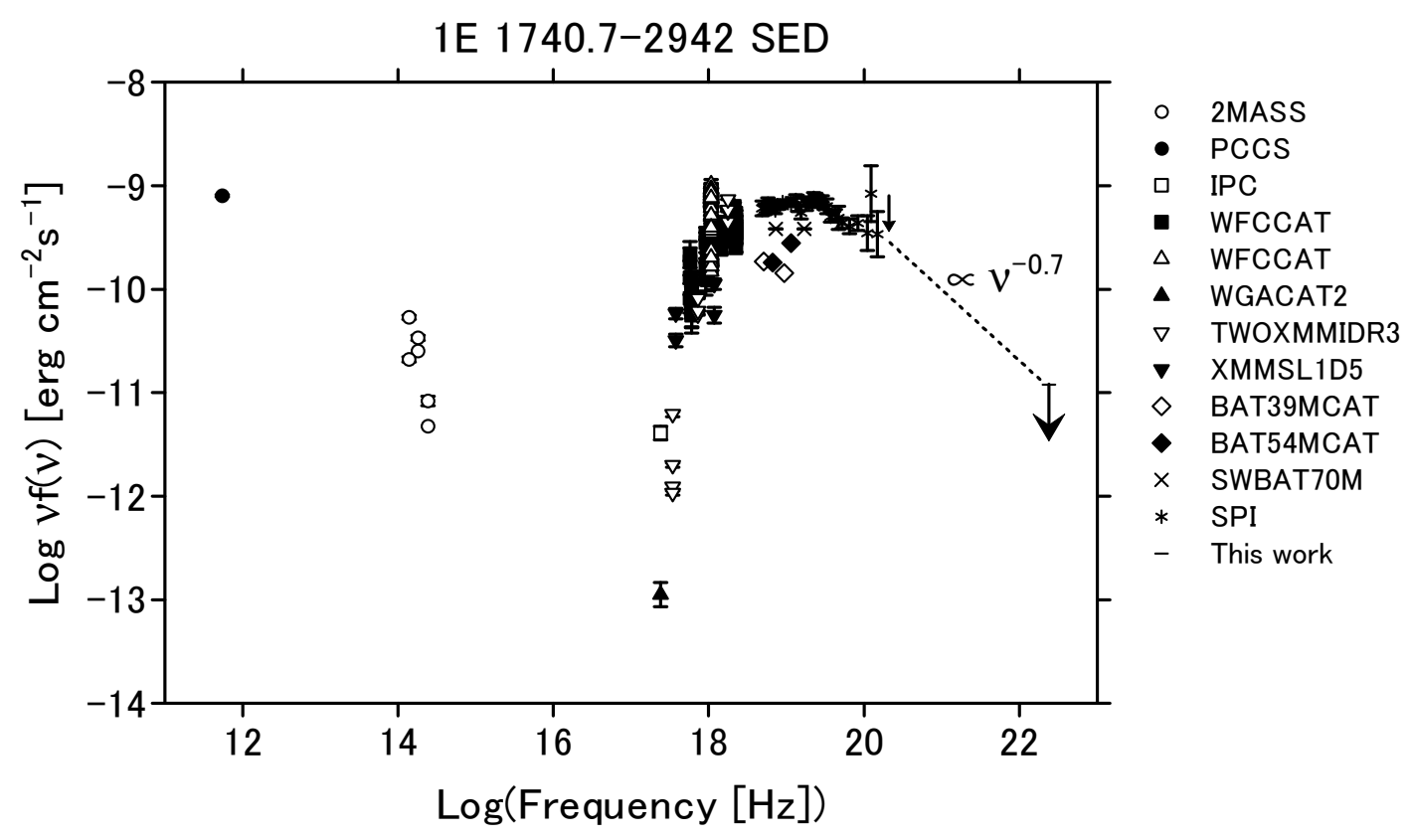

Figure 3: Spectral energy distribution of 1E 1740.7-2942. An upper limit of this work is plotted with other data compiled by ASDC [10] and from INTEGRAL/SPI [5].

\section{References}

[1] Hertz, P., and Grindlay, J. E., ApJ 278, 137 (1984).

[2] Sunyaev, R. et al., A\&A 247, L29-L32 (1991).

[3] Kuznetsov, S. et al., MNRAS, 292, 651 (1997).

[4] Bouchet, L. et al., ApJ 383, L45-L48 (1991).

[5] Bouchet, L. et al., ApJ 693, 1871-1876 (2009).

[6] Bordas, P. et al., ApJ 807, L8 (2015). .

[7] http://fermi.gsfc.nasa.gov/ssc/

[8] Acero, F. et al., arXiv:1501.02003 [astro-ph.HE]

[9] http://swift.gsfc.nasa.gov/results/transients/weak/1E1740.7-2942/

[10] http://tools.asdc.asi.it/

[11] Abdo, A.A. et al., ApJS 188, 405-436 (2010)

[12] Some caveats of the Galactic diffuse models used in the Fermi-LAT analysis are described in: http://fermi.gsfc.nasa.gov/ssc/data/access/lat/BackgroundModels.html 